\title{
Epidemiology, Age, Sex
}

National Cancer Institute

\section{Source}

National Cancer Institute. Epidemiology, Age, Sex. NCI Thesaurus. Code C18768.

The consideration of age and sex as variables in disease incident, transmission, and control. 